\title{
Agent-based automated persuasion with adaptive concessions tuned by emotions
}

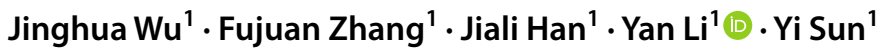 \\ Received: 10 April 2020 / Accepted: 28 February 2021 / Published online: 18 March 2021 \\ (c) The Author(s), under exclusive licence to Springer-Verlag GmbH Germany, part of Springer Nature 2021
}

\begin{abstract}
Human-to-agent automated negotiation has many potentials in a variety of applications. How to design an agent with equivalent persuasion capabilities with its human rivals is the key to the success of such systems but the research on this problem is still at its early stage. With the aim of improving agents' persuasion ability, this paper proposes to construct emotional agents and emotion-dependent persuasion actions in automated negotiation with multiple issues. First, a multi-issue evaluation function adjusted by the rival's reputation is constructed to determine whether emotional persuasion is needed. Then, by applying the Weber-Fechner Law, this paper proposes a method to measure an agent's emotion generated by evaluating the rival's proposal. Persuasion is categorized into four types and an emotion-based method is proposed for an agent to select a persuasion type. The selected persuasion type is further related to updating concessions, so that an agent can make concessions adaptive to both the rival's proposal and the focal agent's emotional state. Moreover, a series of numerical experiments on bilateral negotiation between agents are conducted to illustrate the proposed model and validate its effectiveness in improving negotiation efficiency. Theoretical and practical implications as well as limitations are discussed in the end.
\end{abstract}

Keywords Agent-based negotiation $\cdot$ Persuasion selection $\cdot$ Emotions $\cdot$ Multi-issue $\cdot$ Concessions

\section{Introduction}

Agent-based automated negotiation systems, in particular, the ones with persuasion mechanisms, have recently received increasing attention. Especially 2020's coronavirus pandemic has propelled more and more companies to adopt remote negotiation; agent-based automated negotiation fits this trend. The industry has spent continuous efforts in developing such systems and several pioneers have demonstrated great potentials. For example, RoboNegotiator Inc. provides consumers with an

Yan Li

liyan@cumtb.edu.cn

Jinghua $\mathrm{Wu}$

uwhua@163.com

Fujuan Zhang

1004158048@qq.com

Jiali Han

hanjialiax@126.com

Yi Sun

sy20180825@163.com

1 School of Management, China University of Mining and Technology (Beijing), Beijing, China automated negotiation channel in the auto-dealership industry. ${ }^{1}$ Pactum, an AI-based platform designed to automate personalized negotiations, has been reported to be adopted by Walmart Inc. to automate negotiations with part of its global suppliers. ${ }^{2}$

One of the central challenges to designing such human-toagent automated negotiation systems is to increase the agent's persuasion ability. As a human-delegated software entity, an agent which is constructed to follow preset negotiation protocols automatically negotiates with human rivals. Due to the uncertainty embedded in humans' persuasion behavior, a software agent with the equivalent persuasion ability is needed for effective interactions with human rivals to achieve desirable outcomes (Cao et al. 2015). Towards this end, a number of studies have attempted to endow agents with human's mental states such as emotions, so that these emotional agents are able to emulate human persuasion behavior at an advanced level (Castellanos et al. 2018; Jain and Dahiya 2012).

\footnotetext{
${ }^{1}$ See the official website of RoboNegotiator. https://www.robonegoti ator.com/industries/automobile-dealership/

${ }^{2}$ Fortune 50 Company to use Pactum AI for Supplier Negotiations to Create Increased Value for Suppliers and Customers. https://www. pactum.com/Pactum_press_release_2.pdf
} 
Emotions are a significant ingredient of human life and have been shown to deeply interconnect with cognition in affecting decisions (Maria and Zitar 2007). Psychological evidence has shown that emotions actually can help humans to deal with decision-making more effectively (Meyer 2006). Emotions also play a positive role in human-conducted negotiation (Basheer et al. 2015; Mehrabian 1996; Paradeda et al. 2017). Emotions as a comprehensive response to external stimuli (i.e., rivals' proposals) affect a negotiator's persuasion strategies and tactics, in turn influencing a rival's negotiation behavior. As such, skilled negotiators need to attune to both their own emotions and their rivals' (Leary et al. 2013). Therefore, it is of great value to equip agents with emotions to achieve a higher intelligence level.

Measuring emotions and converting emotions into persuasion actions are two tasks to create emotional agents. The existing research has done some initial efforts towards investigating the effects of basic emotions including happiness, sadness, anger, and fear on agent-based negotiation. However, besides those basic ones, emotions in fact include a spectrum of subtypes (Moerland et al. 2018). The slight difference between adjacent subtypes of emotions reflects a human's distinct perception of the stimuli and may inflict unignorable effects on decision outcomes. Thus, it is of value to quantify emotions to reflect this characteristic. The first objective of this research is thus to design such a quantification method for emotions.

Moreover, the dynamic interactions between agents' emotions and persuasion actions, e.g., making concessions, have been less researched. The existing literature mainly deals with static rules for making concessions, such as pre-fixed concession steps (Chen and Li 2010). Some studies propose dynamic concession strategies but they are mostly adjusted based on explicit clues such as the opponents' proposals (Van Kleef et al. 2006; Subagdja et al. 2019; Louta et al. 2008). The interaction mechanisms via some implicit clues such as emotions just begin to get research attention.

Emotion-driven persuasion may also have strategic effects on negotiation. A large body of research has spent ceaseless efforts in improving a software agent's computational ability with the objective of maximizing the negotiation outcomes. However, the software agent and the human delegator constitute a strategic delegation relationship beyond a principal-agent one, because of the delegated agent's competitive interactions with rivals. The strategic delegation literature, dating back to Vickers (1985) and Fershtman and Judd (1987), shows that by delegating strategic decisions to the agent who does not behave as a rational maximizer, the delegator can obtain better outcomes. By this delegation, a credible competitive commitment is revealed to the rivals and can alter the rivals' behavior in a favorable direction (Sengul et al. 2012). In the same vein, by equipping an agent with emotion-driven concessions, a commitment is shown to the human rival that the agent's persuasion actions will be mixed with emotional and rational elements. This commitment may be helpful for reducing goal conflicts and promote the human rival to compromise with the agent towards mutually satisfactory agreements (Ramezani et al. 2011; Martinovski 2010). Thus, the second objective of this paper is to model persuasion and concessions dependent on an agent's emotional states (i.e., emotional persuasion).

Our research makes the following contributions to agentbased automated negotiation. Firstly, we propose an emotion-adjusted concession model which equips an agent with more human-like persuasion behavior; as such, more appropriate concessions can be made, leading to a quicker negotiation success. Secondly, we employ the Weber-Fechner Law to establish a correspondence between an agent's emotion and its evaluation of a rival's proposal; accordingly, the emotion can be finely calibrated to capture a gradual change in an agent's perception of the opponent's proposal. Thirdly, we propose an emotion-based approach to select the type of persuasion; as a result, agents can switch persuasion actions driven by their inner states and thereby achieve a greater autonomous degree.

The rest of the paper is organized as follows. Section 2 reviews the related work. Section 3 constructs a multi-issue evaluation function, quantifies the emotion, proposes an emotion-based method for persuasion selection, establishes the emotion-adjusted concession model, and designs a negotiation protocol. Section 4 conducts a series of numerical experiments to illustrate the negotiation procedures, to make sensitive analyses to show negotiation results relative to some parameters, and to justify our proposed model's advantages by nested comparisons. This paper is concluded in Sect. 5.

\section{Literature review}

Our research is related to the following two streams of research on agent-based automated negotiation, i.e., increasing the anthropomorphic level of agents and improving automated negotiation efficiency.

The research on increasing the anthropomorphic level of agents attempts to endow agents with anthropomorphic characteristics, so that agent-based automated negotiation can be more human-like. Some research pointed out that fairness, a factor concerned by humans in interactions with others, can affect the persuasion and speed up the negotiation process (Whitford et al. 2013). Emotions are another human's inherent trait paid attention to by the research on agent-based negotiation. Emotions can help agents adjust their negotiation strategies and concessions, enriching agents' intelligent behavior and improving the anthropomorphic level of agents. Maria and Zitar (2007) discussed roles of emotions 
in agent-based systems and displayed the advantages of the systems with emotions relative to the ones without emotions. A batch of research paid attention to the representation of emotions. For example, the KARO logic was employed to represent basic emotions (Meyer 2006). In addition to the studies that focused on modeling an individual agent's emotions, another batch of research studied emotions in interactions among agents. Santos et al. (2011) introduced emotions into agent-based group decision support systems. Ruijten et al. (2016) adopted persuasive techniques to help agents express their emotions. Salgado and Clempner (2018) proposed an agent-based adaptive emotional framework, which modeled emotions as states in a Markov chain and measured emotional states among interacting agents.

Research on incorporating emotions into agent-based negotiation has been at the initial stage and the existing literature is centered with identifying basic types of emotions that may have effects on negotiation. Our work advances this stream of research by proposing a method to quantitatively connect an agent's emotion to the stimuli (a rival's proposal), so that a wide spectrum of emotions can be measured. Moreover, we also establish the correspondence between emotions and persuasion, accordingly making it possible to characterize emotion-dependent persuasion actions.

Improving the negotiation efficiency is the ongoing focus of the research on automated negotiation. This stream of research aims to design various ways to achieve this purpose. Xue et al. (2009) proposed a relative-entropy based method to improve agent-based negotiation efficiency in a construction supply chain. Wong and Fang (2010) proposed a multi-intelligent agent protocol which can increase negotiation efficiency in complex multilateral and multi-issue negotiations. Kowalczyk et al. (2004) designed collaborative scheduling in an adaptive network to enhance negotiating agents' efficiency. Some research designed an iterative process for automated negotiation, improving agents' negotiation efficiency in a dynamic environment. By introducing agent-based automated negotiation into a shipping supply chain, Wang et al. (2017) displayed that automated negotiation improved both parties' benefits. Hajimiri et al. (2014) designed an intelligent negotiator agent for negotiating bilateral contracts of electricity energy, effectively overcoming the time constraint. Cao et al. (2015) established a time-dependent strategies so that agents could cope with the changing negotiation situation to achieve a higher negotiation success rate. Yu and Wong (2015) designed an agentbased negotiation model for the supplier selection with multiple products and by exploiting the synergy effects among products, the designed model could improve the procurement efficiency.

The existing studies tried their best to improve the rationality level of agents to improve the efficiency of agentbased negotiation. By contrast, our research shows that the negotiation efficiency can also be improved by increasing the anthropomorphic level of agents.

\section{Model description}

Agent-based emotional persuasion refers to the type of agent-based automated persuasion which considers the effects of emotions on an agent's persuasion behavior. Emotions are the response to the external stimuli or events that are important to the focal decision-maker (Creed et al. 2014; Mian and Oinas-Kukkonen 2016). By integrating emotions into an agent's persuasion, agent-based emotional persuasion makes an agent able to switch its persuasion actions in terms of the agent's perceptions of dynamic negotiation situations.

We construct a model to characterize an agent's emotions and its effects on the agent's automated negotiation behavior, shown in Fig. 1. Each agent is constructed to have four processing modules. The first module, the evaluation of a proposal, calculates an agent's utility from accepting the rival's proposal and determines whether emotional persuasion is needed. If the answer is yes, the module of emotion generation produces an agent's emotion in terms of the rival's proposal. Then, the generated emotion is mapped into a specific type of persuasion in the module of the selection of a persuasion type. A concession adjusted coefficient corresponding to each persuasion type is selected and used in the last module to compute the agent's concessions and the new proposal is formed consequently.

\subsection{An agent's evaluation of a rival's proposal}

In decision-making, a decision-maker's utility measures his/her satisfaction of a proposal. The higher the utility, the higher the decision-maker's satisfaction of the proposal, and vice versa. Thus, a utility function can help a decisionmaker to choose the next action based on his/her level of satisfaction.In the field of agent-based automated negotiation, a negotiation often deals with multiple issues such as the price, the quality, and the delivery time; therefore, an agent mainly uses a multi-issue utility function to appraise a proposal. In this research, this multi-issue utility function is constructed by the weighted sum of the evaluation of each negotiating issue contained in a proposal.

A proposal made by agent $i(i=1,2, \ldots, l)$ in the $n$th round of emotional persuasion is denoted as a set, i.e., $P_{i}^{(n)}=\left\{t_{i}^{(n)}(T), T \in \Theta \mid S_{i}^{(n)}, e_{i}^{(n)}, \varphi_{i}\left(r_{j}\right), j \neq i\right\}$, where $\Theta$ is the set of all the negotiating issues, $t_{i}^{(n)}(T)$ represents the proposed value of an issue $T, S_{i}^{(n)}$ is the selected type of persuasion, and $e_{i}^{(n)}$ is the emotion. Besides, $\varphi_{i}\left(r_{j}\right)$ measures the effects of a rival agent $j$ 's $(j \neq i)$ reputation and it is a function of agent $j$ 's reputation level denoted as $r_{j} \in[0,1]$. It is assumed that an agent's reputation level keeps constant 
Fig. 1 The model framework for the proposed emotional persuasion

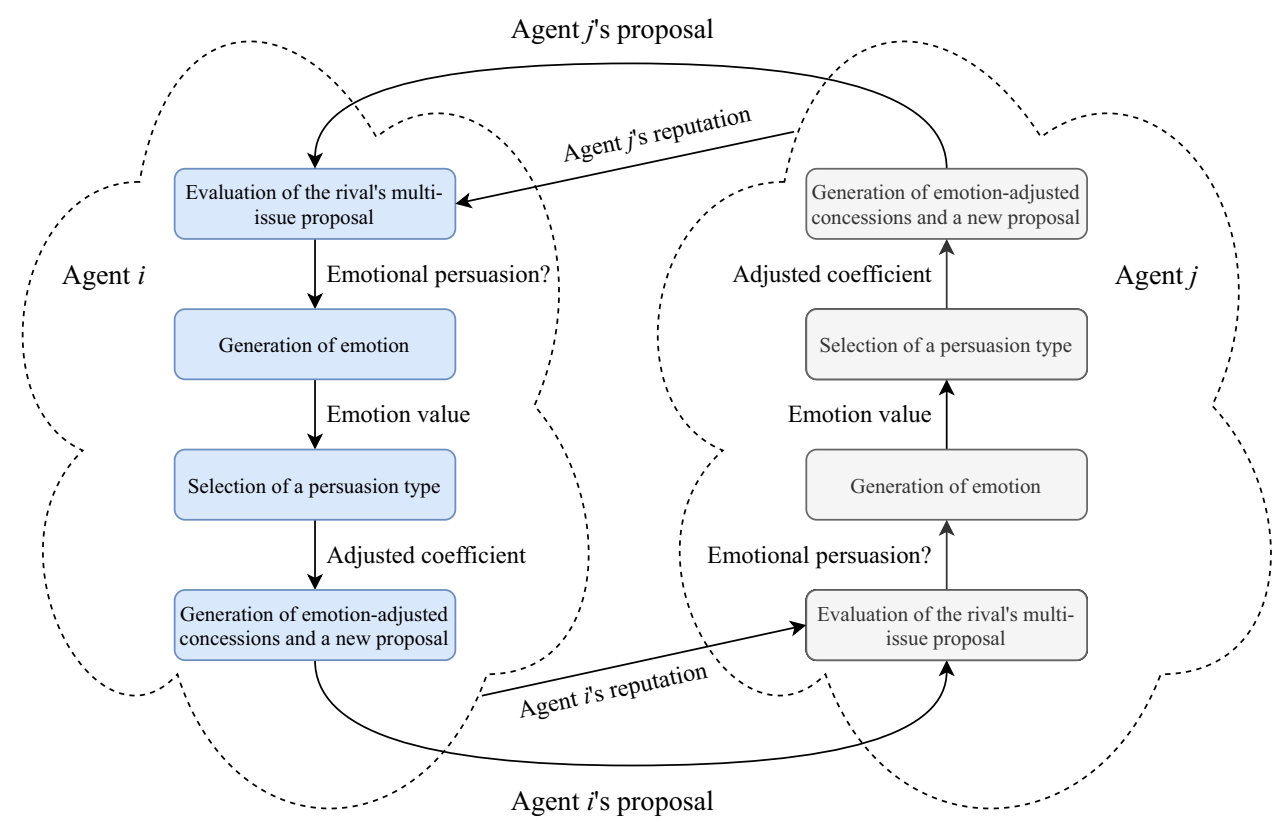

during negotiation and thus $\varphi_{i}\left(r_{j}\right)$ is fixed once the negotiating target is selected.

Both negotiating agents have contrasted views of a negotiating issue. If the buyer agent considers an issue to be a cost-type (i.e., the lower the issue value, the higher the agent's utility), the seller agent will consider this issue to be a benefit-type. Based on the above considerations, we construct the following evaluating function of a negotiating issue.

Assume that agent $i$ evaluates an issue $T$ with a value of $t_{j}$ proposed by agent $j(j \neq i)$. Agent $i$ 's maximum and minimum acceptable values of the issue are $\bar{t}_{i}$ and $t_{i}$, respectively. Agent $i$ 's evaluating function denoted as $f_{i}\left(t_{j}\right) \overline{\text { is then defined }}$ as follows,

$f_{i}\left(t_{j}\right)= \begin{cases}1, & t_{j} \leq \underline{t_{i}} \\ \left(\frac{t_{j}-\bar{t}_{i}}{\overline{t_{i}-t_{i}}}\right)^{2}, & , \underline{t_{i}}<\bar{t}_{j}<\overline{t_{i}} \\ 0, & t_{j} \geq \overline{t_{i}}\end{cases}$

$f_{i}\left(t_{j}\right)= \begin{cases}0, & t_{j} \leq \underline{t_{i}} \\ \left(\frac{t_{j}-t_{i}}{\overline{t_{i}-t_{i}}}\right)^{2}, & t_{i}<\bar{t}_{j}<\overline{t_{i}} \\ 1, & t_{j} \geq \overline{t_{i}}\end{cases}$

Equation (1a) evaluates a cost-type negotiating issue and Eq. (1b) evaluates a benefit-type issue. It is assumed that $\forall T \in \Theta$, if agent $i$ views $T$ as a cost-type issue and agent $j$ views it as a benefit-type, then $\underline{t_{i}}<\underline{t_{j}}$ and $\bar{t}_{i}<\bar{t}_{j}, i \neq j$. This assumption is based on the following considerations. In business negotiation, both proficient negotiating parties will have comparable estimations of the value range of a negotiating issue, since both of them have accumulated sophisticated knowledge and experience about a product/service. On this basis, the seller will shift the range upwards whereas the buyer will shift the range downwards from their own standpoints. The shapes of the evaluating functions are shown in Fig. 2.

An agent's evaluation of a negotiating issue will also be affected by the rival agent's reputation (Akhgar et al. 2014). An agent will give a higher evaluation of the proposal from an opponent with a higher reputation than the one from an opponent with a lower reputation, even if their proposals are same. A good reputation indicates an agent's sound performance and negotiating behavior; the accumulated reputation will deter the agent from doing opportunistic actions in the future.

An agent is assumed to have an acceptable minimum reputation level of a rival party. Let $R_{i j} \in(0,1)$ be agent $i$ 's acceptable minimum reputation level of agent $j(j \neq i)$. When $r_{j}<R_{i j}$, let $\varphi_{i}\left(r_{j}\right)=0$, indicating that agent $j$ 's reputation is too low to have any positive effects on agent $i$ 's evaluation; when $r_{j} \geq R_{i j}$, as $r_{j}$ increases, so does $\varphi_{i}\left(r_{j}\right)$, implying increasing positive effects of agent $j$ 's reputation. The above relationship is depicted by the following equation,

$\varphi_{i}\left(r_{j}\right)=\left\{\begin{array}{ll}0, & 0 \leq r_{j}<\underline{R_{i j}} \\ \underline{r_{j}-R_{i j}}, \underline{1-R_{i j}} & R_{i j} \leq r_{j} \leq 1\end{array}\right.$,

where $i \neq j$ and $\varphi_{i}\left(r_{j}\right) \in[0,1]$. 


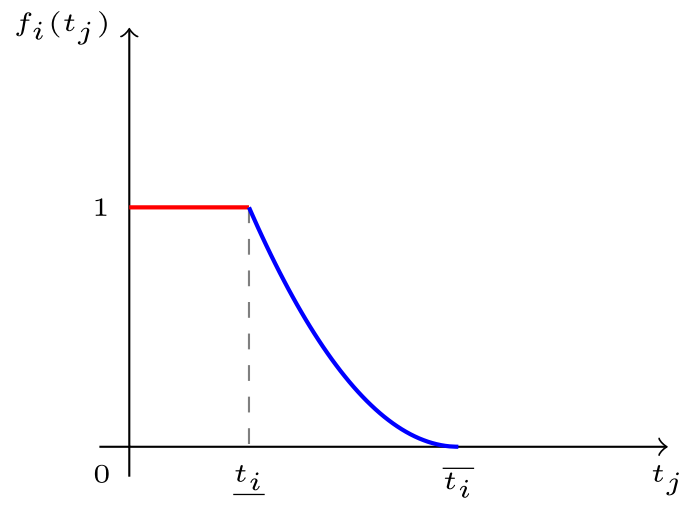

(a) A cost-type issue

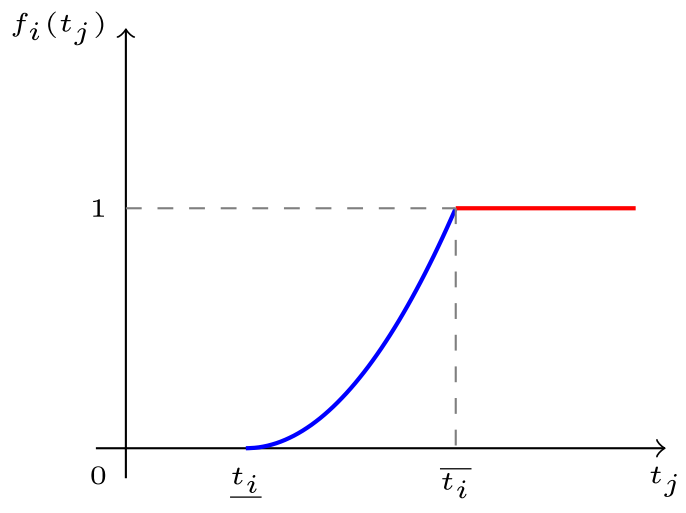

(b) A benefit-type issue

Fig. 2 An agent's evaluating function of a negotiating issue

After all the individual issues have been evaluated, they will be integrated to form the weighted evaluation value (i.e., the total utility). Agent $i$ 's multi-issue total utility function $V_{i}\left(\mathbf{t}_{j}\right)$ is thus formulated as,

$V_{i}\left(\mathbf{t}_{j}\right)=\sum_{T \in \Theta} w_{T_{i}} \varphi_{i}\left(r_{j}\right) f_{i}\left(t_{j}\right)$,

where $\mathbf{t}_{j}$ is a vector of issue values proposed by agent $j, w_{T_{i}}$ is the weight agent $i$ assigns to issue $T$ and $\sum_{T \in \Theta} w_{T_{i}}=1$. Note that $\varphi_{i}\left(r_{j}\right)=0$ actually prohibits agent $i$ from engaging in negotiation with agent $j$ and with $\varphi_{i}\left(r_{j}\right)$ increasing, agent $i$ 's utility is augmented.

For each issue $T$, agent $i$ also has an expected value $t_{i}$. By substituting $\mathbf{t}_{i}$, the vector of the expected issue values of agent $i$, into (3), agent $i$ can calculate its expected total utility $V_{i}^{E}\left(\mathbf{t}_{i}\right)$. If $V_{i}\left(\mathbf{t}_{j}\right)<V_{i}^{E}\left(\mathbf{t}_{i}\right)$, agent $i$ will use emotional persuasion; otherwise, agent $i$ will accept the rival's proposal.

\subsection{Emotion and its generation}

We apply the Weber-Fechner law to formulate the emotion denoted as $e$ as follows,

$e=k \log (1+\Delta x)$

where $k$ is the coefficient and $\Delta x$ is the difference of value rates. In terms of the above formula, $e$ takes a value on $(-\infty, \infty)$, and the larger the absolute value of $e$, the stronger the emotion.

The Weber-Fechner Law, originated in psychology, describes a quantitative relationship between a human's perceived magnitude of a stimulus and the stimulus' physical strength (Kolic and Dyer 2020). It states that a human's perceived magnitude of a stimulus varies with the logarithm of the ratio of the physical magnitude of the stimulus to a threshold that the stimulus has to overcome to be perceived. The Weber-Fechner Law sees a wide application in psychology, such as characterizing a consumer's response to a price discount.

The existing literature points out that the magnitude of the emotion depends on the comparative relationship between a stimulus and some reference against which the stimulus is evaluated (Frijda 1988). Some prevailing mental states and expectation can be used as the reference. In persuasion, an agent usually has the expected value of each negotiating issue and will compare the rival's proposed value against its expected one. Here, the rival's proposed value is the external stimulus and the focal agent's expected value constitutes the reference. Thus, the comparison result determines the magnitude of the focal agent's generated emotion.

In this research, the coefficient $k$ in Eq. (4) is measured by the weight $w_{T_{i}}$. The set of the weights of all the issues denoted as $W$ reflects an agent's preferences over the attributes of a product. The larger the weight of an issue, the more stronger emotion will be generated from evaluating the issue.

All the issues fall into either a benefit-type or a cost-type dependent on an agent's standpoint. The difference of value rates $\Delta x$ is contextualized by the difference of issue values, i.e., $\Delta t$, which can be calculated via the following formula,

$\Delta t=\left\{\begin{array}{l}\frac{t_{o}-t_{f}}{t_{f}}, \text { a benefit-type issue } \\ \frac{t_{f}-t_{o}}{t_{f}}, \text { a cost-type issue }\end{array}\right.$

where $t_{f}$ is the focal agent's expected value of a negotiating issue and $t_{o}$ is the opponent's proposed value of the same issue. The larger the difference between the focal agent's expected value and the opponent's proposed value, the larger the absolute value of the emotion. 
Applying Eq. (5) to every issue and taking the sum, we can obtain agent $i$ 's emotion as follows ${ }^{3}$,

$e_{i}=\sum_{T \in \Theta} w_{T_{i}} \log \left(1+\Delta t_{i}\right)$

\subsection{The selection of persuasion types}

The previous research has classified agent-based persuasion into multiple types. For example, Amgoud and Prade (2004) classified agent-based persuasion into threat, reward, explanation, and appeal type. Using the theory of interpersonal persuasion, Sun et al. (2014) divided the agent-based persuasion into complaint, explanation, and analogy type. With reference to those studies, agent-based persuasion in this paper are divided into the following four types,

* The complaint type of persuasion refers to that an agent complains about its proposal because of the proposal's failure to meet the other agent's expectations;

* The explanation type of persuasion refers to that an agent explains its proposal and expounds its reasons to the other agent during persuasion;

* The analogy type of persuasion refers to that an agent displays the merits of its proposal by comparing its proposal with its opponent's;

* The threat type of persuasion refers to that an agent forces the other agent to make concessions via commination.

The selection of persuasion is an important step to enable an agent's persuasion ability. A group of research has been devoted to designing various criteria in accordance with which a type of persuasion will be selected. For instance, the severity, the strength of persuasion and time have been used for selection (Kraus et al. 1998; Monteserin and Amandi 2013). We make a contribution to this strand of research by proposing that an agent's emotion can be used as a factor to determine the persuasion selection. Different magnitudes of emotions generally lead to different persuasion behavior (Bradley and Lang 1994; Wang et al. 2015). In general, as the absolute value of the emotion gets larger, the more aggressive type of persuasion will be adopted, and vice versa. In particular in negotiation between supply chain members, this emotion-driven persuasion selection is also intertwined with the relationship between the negotiating parties. For example, in a supply chain where the buyer has more market power, the agent representing the buyer will

\footnotetext{
${ }^{3}$ For the case where $1+\Delta t_{i} \leq 0, e_{i}$ is set to be $-\infty$. It means the rival's proposed issue value is way below an agent's expectation and the agent will accordingly have strongly negative emotion.
}

select the type of persuasion in the order of threat, analogy, explanation, and complaint, whereas the agent representing the seller will select the type of persuasion in the order of complaint, explanation, analogy, and threat, as the negative emotion weakens.

\subsection{Emotion-adjusted concessions}

This research adopts a decision theoretic approach to update an agent's proposal (Sycara 1990). That is, an updated value of an issue depends on the current state and the concessions made in the previous round. Each agent has a base concession for each negotiating issue according to its preferences. The base concession of an issue is inversely related to the importance of the issue.

As is shown in the previous subsection, an agent's emotions will affect the agent's selection of the persuasion type. These types of persuasion further have varied effects on the concessions that an agent is willing to make. Taking this point into consideration, the base concession will be adjusted by the type of persuasion selected by an agent. The adjusted value is called the emotion-adjusted concession.

An agent will assign an adjusted coefficient to each type of persuasion and the more aggressive a type of persuasion, the larger the corresponding adjusted coefficient. Assume that agent $i$ sets a base concession denoted as $s_{i}$ for an issue $T, T \in \Theta$ with a value of $t_{i}$. The adjusted coefficient of a persuasion type is denoted as $\alpha_{i}, \alpha \in \Omega$, where $\Omega$ denotes the set containing all the four types of persuasion. The emotionadjusted concession will be $\alpha_{i} \times s_{i}$. Then, agent $i$ will update the issue $T$ s value via the following formula,

$\tilde{t}_{i}=t_{i}+\alpha_{i} \times s_{i}$,

where $\tilde{t}_{i}$ is the updated issue value.

\subsection{The negotiation protocol}

We design a negotiation protocol for fulfilling the proposed emotional persuasion. Figure 3 shows the flow chart of the designed negotiation protocol and the specific procedures are explained as follows.

1. At the beginning, both negotiating agents set their individual maximum acceptable rounds of persuasion and the minimum of the two is set as the maximum number of persuasion rounds denoted as $N_{\max }$. Then, both agents fill in the transaction information such as the acceptable lowest and highest values of a negotiating issue. Both agents' reputation levels are shown to each other.

2. An bargaining interval is then automatically computed according to the submitted transaction information. If the bargaining interval does not exist, it is unnecessary 
Fig. 3 The flow chart of the negotiation protocol

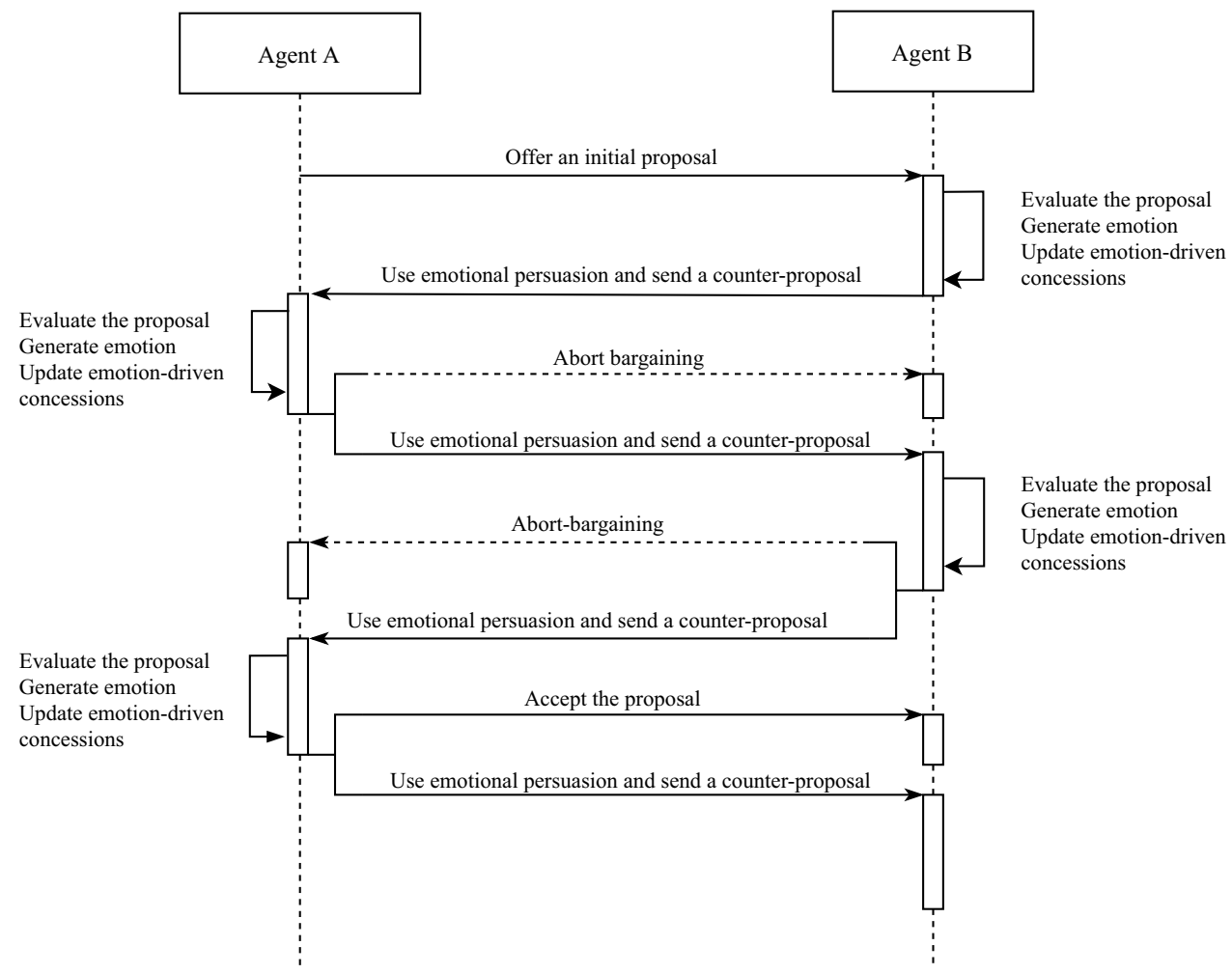

for the emotional persuasion to proceed and the negotiating channel will be closed. If it exists, both agents will proceed the negotiation.

3. Each agent will first use its expected value of each negotiating issue and the other agent's reputation level to calculate its expected total utility via Eq. (3) and will use it as a threshold for comparison.

4. Next, both agents in turn send their proposals or counter-offers. In each round of negotiation, each agent will evaluate every issue in the other agent's proposal and then calculate its total utility from accepting the proposal according to Eq. (3).

5. An agent will compare the calculated total utility with its threshold. If the difference is beyond one agent's expectation and each issue value proposed by the other agent is within the agent's acceptable range, the focal agent will accept the proposal and the persuasion finishes. However, if the difference is below the agent's expectation, or any proposed issue value is out of the agent's acceptable range, the focal agent will change to emotional persuasion. The agent will select a type of persuasion according to its current emotion.

6. Under the selected type of persuasion, an agent will choose the corresponding concession adjusted coefficient and then update the issue value via Eq. (7). If the updated issue value is out of the agent's value range, the boundary value will be automatically used. After that, the updated issue values as the agent's counter-offer for this round of persuasion will be sent to the other agent.

7. Procedures (4) through (6) will keep repeating themselves until the emotional persuasion succeeds, fails, or the number of negotiation rounds exceeds $N_{\max }$.

\section{Numerical experiments}

This section conducts a series of numerical experiments to illustrate the proposed concession model and the negotiation protocol of emotional persuasion, to investigate the negotiation results with parameter value changing, and to show the advantages of the proposed model relative to some base models. It is infeasible to consider all of the possibilities and thus we follow the previous research's approach by considering a representative setting in which the proposed model can be assessed. If the proposed model results in better negotiation outcomes than those without the model in the same setting, the effectiveness of the proposed model can be claimed (Cao et al. 2015; Zhang et al. 2011).

\subsection{Experiment settings}

We consider a bilateral negotiation between a buyer (Agent $A$ ) and a seller (Agent $B$ ) in a supply chain engage in negotiation about some product according to the proposed negotiation protocol shown in Fig. 3. Though the proposed model 
Table 1 Agent A's values and weights of the issues

\begin{tabular}{lllll}
\hline & Price & Quality & Delivery time & Threshold \\
\hline Weight & 0.3 & 0.5 & 0.2 & \\
Expected value & 5 & 8 & 5 & 0.102 \\
Initial proposal & 3 & 9 & 3 & 0.484 \\
Minimum value & 3 & 7 & 3 & \\
Maximum value & 7 & 10 & 6 & \\
\hline
\end{tabular}

aims to improve human-to-agent negotiation, the research on agent-to-agent automated negotiation is valuable for validating the effectiveness of the proposed model (Pan et al. 2013).

Suppose both agents care about the price, the quality, and the delivery time, the main attributes of a product. That is, the set of the negotiating issues is $\Theta=$ \{price, quality, delivery time $\}$. The three negotiating issues have different units, for instance, delivery time is measured by "day" and price is measured by a currency and thus for comparability and simplicity, all the three attributes are graded on a discrete set $\{1,2,3, \ldots, 10\} .{ }^{4}$ The larger the original value of an issue, the larger the graded value of the issue. Agent A's reputation value is $r_{A}=0.8$ and its acceptable minimal reputation value of a rival is $R_{A B}=0.7$; Agent $B$ 's reputation value is $r_{B}=0.9$ and its acceptable minimal reputation value of a rival is $R_{B A}=0.6$.

An agent firstly calculates the effects of a rival's reputation. In Agent B's view, since Agent A's reputation value is larger than its acceptable minimum value, the effect of Agent $A$ 's reputation is calculated as $\varphi_{B}\left(r_{A}\right)=\frac{r_{A}-R_{B A}}{1-R_{B A}}=\frac{0.8-0.6}{1-0.6}=0.50$; similarly, Agent $A$ calculates the effect of Agent $B$ 's reputation as $\varphi_{A}\left(r_{B}\right)=\frac{r_{B}-\underline{R_{A B}}}{1-R_{A B}}=\frac{0.9-0.7}{1-0.7}=0.67$.

Agent $A$ sets the maximum rounds of persuasion to be 50 and this number set by Agent $B$ is 60 . The minimum of the two is selected as the maximum number of rounds of persuasion, i.e., $N_{\max }=50$.

Agents' initial proposals, the weights of the negotiating issues, the adjusted coefficients, the base concessions, and the selection rules of the persuasion type are displayed from Tables 1, 2, 3, 4 and 5 .

\subsection{An illustration of the proposed emotional persuasion}

Agent A (the buyer) is assumed to start the negotiation. The negotiation started by a seller will lead to similar results.
Table 2 Agent B's values and weights of the issues

\begin{tabular}{lllll}
\hline & Price & Quality & Delivery time & Threshold \\
\hline Weight & 0.5 & 0.2 & 0.3 & \\
Expected value & 7 & 7 & 6 & 0.032 \\
Initial proposal & 9 & 6 & 7 & 0.194 \\
Minimum value & 6 & 4 & 4 & \\
Maximum value & 10 & 9 & 9 & \\
\hline
\end{tabular}

Table 3 The concession adjusted coefficient of each persuasion type, $\alpha \in \Omega$

\begin{tabular}{lllll}
\hline & $\begin{array}{l}\text { Complaint } \\
\text { type }\end{array}$ & $\begin{array}{l}\text { Explana- } \\
\text { tion type }\end{array}$ & Analogy type & Threat type \\
\hline Agent $A$ & 4 & 3 & 2 & 1 \\
Agent $B$ & 1 & 2 & 3 & 4 \\
\hline
\end{tabular}

Table 4 The base concessions of the issues

\begin{tabular}{llll}
\hline & Price & Quality & Delivery time \\
\hline Agent $A$ & 0.2 & 0.1 & 0.3 \\
Agent $B$ & 0.15 & 0.3 & 0.2 \\
\hline
\end{tabular}

Table 5 The selection rules of persuasion types

\begin{tabular}{lll}
\hline Range of the emotion $^{\mathrm{a}}$ & $\begin{array}{l}\text { The type of persua- } \\
\text { sion adopted by } \\
\text { Agent } B\end{array}$ & $\begin{array}{l}\text { The type of persua- } \\
\text { sion adopted by } \\
\text { Agent } A\end{array}$ \\
\hline$(-\infty,-0.30]$ & Complaint type & Threat type \\
$(-0.30,-0.15]$ & Explanation type & Analogy type \\
$(-0.15,-0.08]$ & Analogy type & $\begin{array}{l}\text { Explanation type } \\
\text { Complaint type }\end{array}$ \\
\hline
\end{tabular}

a The cut-off values are computed for this experiment setting and they should be contextualized with the change of settings

In the first round: Agent $A$ initially sends a procurement proposal to Agent $B$. The initial proposal includes the grades of all the negotiating issues. The grades of the price, the quality, and the delivery time are 3,9, and 3, respectively. At this stage, Agent $A$ does not know whether its proposal will be accepted by Agent B; therefore, Agent $A$ will not use emotional persuasion. After receiving the proposal, Agent $B$ evaluates the proposal and the results are shown in Table 6.

In this round of persuasion, Agent $B$ evaluates each issue in terms of Eqs. (1a) and (1b) and calculates its total utility from accepting Agent A's proposal via Eq. (3),

\footnotetext{
${ }^{4}$ In practice, the comparability among the attributes of a product can be obtained by normalization. Please refer to Yu and Wong (2015).
} 
Table 6 Agent B's evaluation of Agent A's proposal in the first round of persuasion

\begin{tabular}{llll}
\hline & Price & Quality & Delivery time \\
\hline Type of the issue & Benefit type & Cost type & Benefit type \\
Agent A's proposal & 3 & 9 & 3 \\
Evaluated value & 0 & 0 & 0 \\
Weight of an issue & 0.5 & 0.2 & 0.3 \\
\hline
\end{tabular}

Table 7 Agent A's evaluation of Agent B's counter-offer in the first round

\begin{tabular}{llll}
\hline & Price & Quality & Delivery time \\
\hline Type of the issue & Cost type & Benefit type & Cost type \\
Agent B's proposal & 9 & 6 & 7 \\
Evaluated value & 0 & 0 & 0 \\
Weight of an issue & 0.3 & 0.5 & 0.2 \\
\hline
\end{tabular}

$$
\begin{aligned}
V_{B} & =\sum_{T \in \Theta} w_{T_{B}} \varphi_{B}\left(r_{A}\right) f_{B}\left(t_{A}\right) \\
& =(0 \times 0.5+0 \times 0.2+0 \times 0.3) \times 0.5=0 .
\end{aligned}
$$

This total utility is way below Agent B's expected one and thus Agent $B$ will decline Agent A's proposal and start emotional persuasion. To achieve that, Agent $B$ needs to calculate its emotion according to (6),

$$
\begin{aligned}
e_{B}= & 0.5 \times \log \left(1+\frac{3-7}{7}\right)+0.2 \times \\
& \log \left(1+\frac{7-9}{7}\right)+0.3 \times \log \left(1+\frac{3-6}{6}\right)=-0.304 .
\end{aligned}
$$

The above result shows that the emotion is negatively strong; thus, according to the selection rules of persuasion in Table 5, Agent B will choose the complaint-type of persuasion to compel Agent $A$ to adjust issue values in the next round. Meanwhile, Agent $B$ initiates its own proposal shown in Table 2.

Then, Agent $A$ evaluates the counter-offer of Agent $B$ and the evaluated results are shown in Table 7 ,

Agent A's total utility from accepting Agent B's counteroffer can be computed as,

$$
\begin{aligned}
V_{A} & =\sum_{T \in \Theta} w_{T_{A}} \varphi_{A}\left(r_{B}\right) f_{A}\left(t_{B}\right) \\
& =(0 \times 0.3+0 \times 0.5+0 \times 0.2) \times 0.67=0 .
\end{aligned}
$$

Table 9 The process of Agent A's persuasion

\begin{tabular}{llllll}
\hline \multirow{2}{*}{\begin{tabular}{l} 
Rounds \\
\cline { 3 - 4 }
\end{tabular}} & $\begin{array}{l}\text { Received type } \\
\text { of persuasion }\end{array}$ & \multicolumn{2}{l}{ Proposal } & Total utility \\
\cline { 3 - 5 } & & Price & Quality & Delivery time \\
\hline 1 & & 3 & 9 & 3 & 0.000 \\
2 & Complaint & 3.8 & 8.6 & 4.2 & 0.000 \\
3 & Explanation & 4.4 & 8.3 & 5.1 & 0.039 \\
4 & Analogy & 4.8 & 8.1 & 5.7 & 0.082 \\
5 & Analogy & 5.2 & 7.9 & $6^{\mathrm{a}}$ & 0.170 \\
6 & Threat & 5.4 & 7.8 & $6^{\mathrm{a}}$ & 0.178 \\
7 & Threat & 5.6 & 7.7 & $6^{\mathrm{a}}$ & 0.187 \\
8 & Threat & 5.8 & 7.6 & $6^{\mathrm{a}}$ & 0.197 \\
\hline
\end{tabular}

${ }^{\text {a }}$ The original value is changed to the maximum value of the negotiating issue acceptable to Agent $A$

Clearly, there is a gap between Agent A's calculated total utility and its expected one. Therefore, Agent $A$ rejects Agent $B$ 's counter-offer and starts its emotional persuasion. Agent $A$ 's emotion is,

$$
\begin{aligned}
e_{A}= & 0.3 \times \log \left(1+\frac{5-9}{5}\right)+0.5 \\
& \times \log \left(1+\frac{6-8}{8}\right)+0.2 \times \log \left(1+\frac{5-7}{5}\right)=-0.317 .
\end{aligned}
$$

At the moment, Agent A's emotion is negatively strong and according to the selection rules of persuasion, Agent $A$ uses the threat-type of persuasion. Based on Agent A's base concessions of the issues in Table 4 and the adjusted coefficient corresponding to the complaint-type in Table 3, the adjusted proposal put forward by Agent $A$ via Eq.(7) is shown in Table 8.

In the following rounds of emotional persuasion, the proposed issue values, the selected types of persuasion, the total utility, and the emotion is calculated with the same approach described in the first round; the results are summarized in Tables 9 and 10.

Both Tables 9 and 10 show that from the 6th to the 8th rounds of persuasion, although Agent $A$ 's proposal is within Agent B's acceptable range, Agent $B$ continues with emotional persuasion because Agent $B$ 's calculated total utility does not meet its expected value. Finally, in the 8th round, Agent $B$ 's total utility reaches its expected value and the proposed value of each issue is also within Agent A's acceptable range; thus, the emotional persuasion is completed and the transaction succeeds. At the moment, the agreed grades of
Table 8 Agent A's proposal in the second round

\begin{tabular}{llll}
\hline & Price & Quality & Delivery time \\
\hline Adjusted issue value & $3+0.2 \times 4=3.8$ & $9-0.1 \times 4=8.6$ & $3+0.3 \times 4=4.2$ \\
\hline
\end{tabular}


Table 10 The process of Agent B's persuasion

\begin{tabular}{llllll}
\hline Rounds & $\begin{array}{l}\text { Received type } \\
\text { of persuasion }\end{array}$ & \multicolumn{2}{l}{ Proposal } & Total utility \\
\cline { 3 - 5 } & & Price & Quality & Delivery timen \\
\hline 1 & 9 & 6 & 7 & 0.000 \\
2 & Threat & 8.4 & 6.9 & 6.2 & 0.001 \\
3 & Analogy & 7.95 & 7.8 & 5.6 & 0.009 \\
4 & Explanation & 7.65 & 8.4 & 5.2 & 0.021 \\
5 & Explanation & 7.35 & $9^{\mathrm{a}}$ & 4.8 & 0.029 \\
6 & Complaint & 7.2 & $9^{\mathrm{a}}$ & 4.6 & 0.030 \\
7 & Complaint & 7.05 & $9^{\mathrm{a}}$ & 4.4 & 0.031 \\
8 & Complaint & 6.9 & $9^{\mathrm{a}}$ & 4.2 & 0.032 \\
\hline
\end{tabular}

${ }^{\text {a }}$ The original value is changed to the maximum value of the negotiating issue acceptable to Agent $B$

the price, the quality, and the delivery time are $6.9,9$, and 4.2 , respectively.

Figure 4 shows the trends of Agent A's and Agent B's proposed value of each issue. It can be seen that with persuasion proceeding, both agents' proposed values of cost-type issues increase. For Agent A, the buyer, the price and the delivery time are the cost-type issues, whereas for Agent $B$, the seller, the quality is the cost-type issue. When the values increase to the maximum acceptable levels of both agents, they stop increasing. For example, Agent A's proposed value of the delivery time is increased to 6 , the maximum value of this issue acceptable to Agent A, at the 4th round and then stops increasing; Agent B's proposed value of the quality levels at 9 , the maximum value of this issue acceptable to Agent $B$, from the 5 th round. Whereas, the proposed values of the benefit-type issues decrease. For Agent A, the quality is such an issue and for Agent B, this type of issues includes the price and the delivery time.

Figure 5 compares both agents' emotions and the selected types of persuasion in each round. It can be seen that at the beginning, both agents' emotions are quite large negative numbers. That is because an agent's total utility from accepting a proposal by the opponent is way below the focal agent's expectation. This situation leads to the adoption of emotional persuasion. At this moment, both agents will select the type of persuasion with a great persuasive strength. That is, Agent A selects the threat type and Agent B selects the complaint type. As the persuasion proceeds, both agents' negative emotions are weakened and correspondingly the type of persuasion with a mild persuasive strength is in turn selected. This trend implies that each agent's total utility from accepting the opponent's proposal in every round is approaching the focal agent's expected value gradually.

\subsection{Sensitive analysis}

The above illustrative example shows that such parameters as the reputation level and the adjusted coefficient will affect an agent's persuasion behavior. Once a target partner
Fig. 4 Both agents' proposed value of each issue
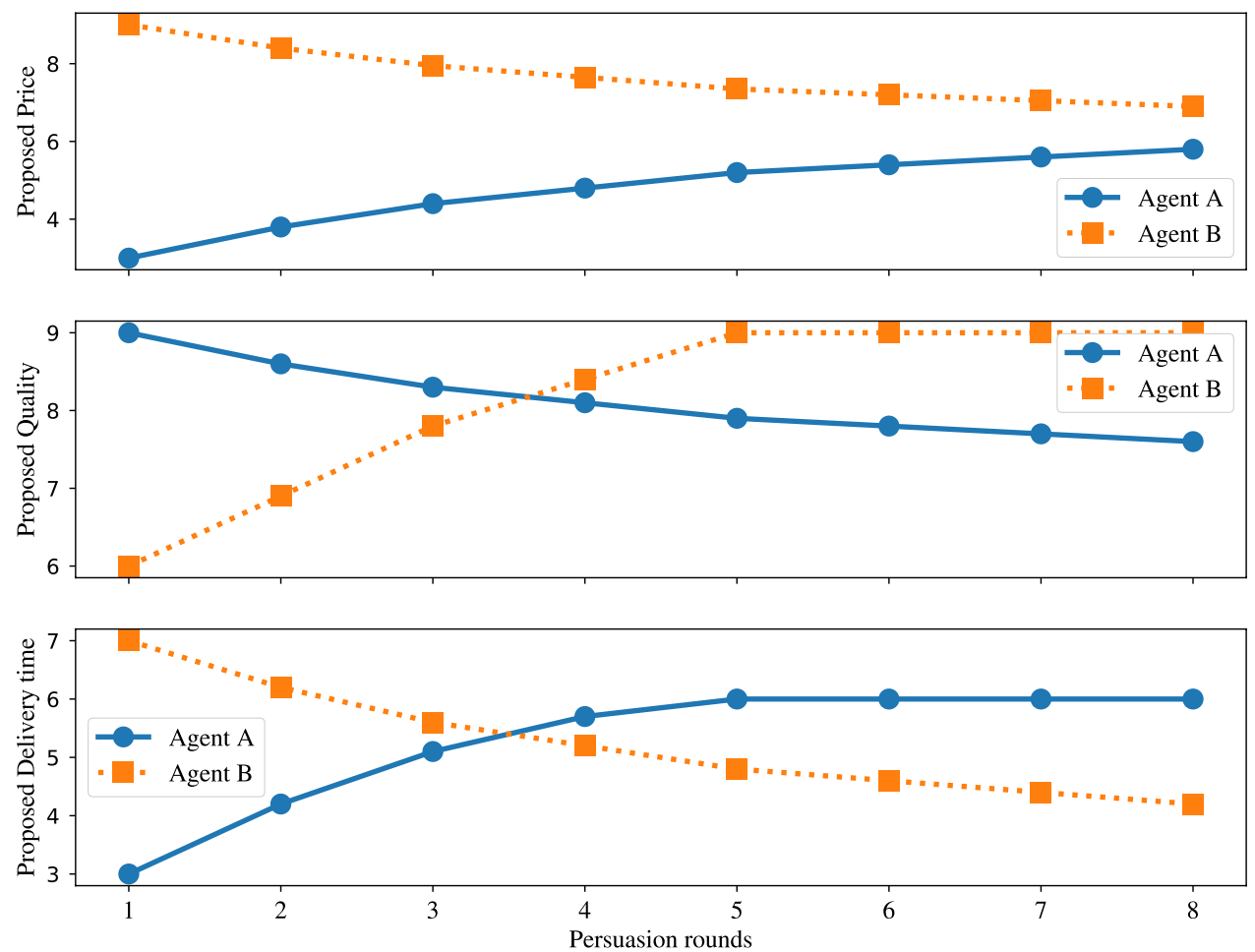


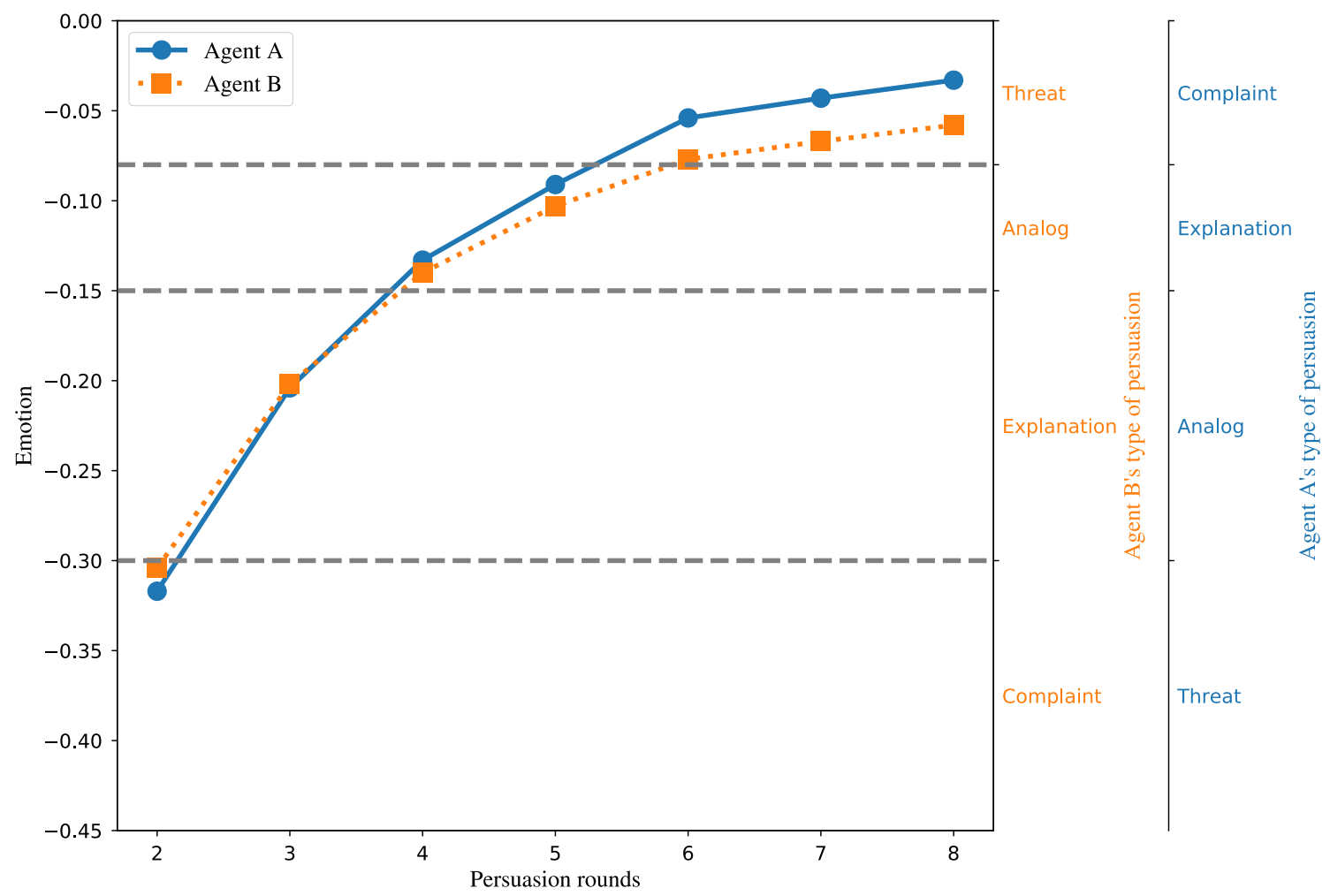

Fig. 5 Both agents' emotion and selected types of persuasion

is selected, the effects of the reputation level will be fixed. Thus, we investigate how negotiation results will be changed with the adjusted coefficient. Table 11 sets the values of the adjusted coefficients for both agents.

By allowing adjusted coefficient $\alpha$ 's to choose values from the set of pairs, we can display the effects of this coefficient on both agent's utilities in each round and the results are shown in Figs. 6 and 7.

Both figures display that as persuasion proceeds, both agents' total utilities are increased. This trend implies that the proposed emotional concession model is generally able to promote negotiation towards a favorable direction. Moreover, the increase in the adjusted coefficient speeds up each agent's total utility's improvement, leading to less time to reach an agreement.

\subsection{Comparisons}

In order to demonstrate the advantages of the proposed emotion-adjusted concession model, a series of nested model comparisons were conducted. First, the proposed model is compared with the model that considers neither emotion nor reputation. Second, the proposed model is compared with the model that considers emotion but reputation. Tables 12 and 13 show the persuasion processes considering neither emotion nor reputation.
Tables 14 and 15 show the persuasion processes considering emotion but reputation.

Those comparisons show that our proposed model have advantages in the following two ways. First, the proposed model considering both emotion and reputation generally leads to a faster convergence in persuasion. For example, both agents in our proposed model reach an agreement within 8 rounds, whereas, they need 10 rounds to reach an agreement in the model considering neither emotion

Table 11 The pairs of concession adjusted coefficients for each persuasion type

\begin{tabular}{llllll}
\hline$\alpha$ & Agents & $\begin{array}{l}\text { Com- } \\
\text { plaint } \\
\text { type }\end{array}$ & $\begin{array}{l}\text { Explana- } \\
\text { tion type }\end{array}$ & Analogy type & Threat type \\
\hline$\alpha^{(1)}$ & Agent A & 4 & 3.5 & 3 & 2.5 \\
& Agent B & 2.5 & 3 & 3.5 & 4 \\
$\alpha^{(2)}$ & Agent A & 4.5 & 4 & 3.5 & 3 \\
& Agent B & 3 & 3.5 & 4 & 4.5 \\
$\alpha^{(3)}$ & Agent A & 5 & 4 & 3 & 2 \\
& Agent B & 2 & 3 & 4 & 5 \\
$\alpha^{(4)}$ & Agent A & 6 & 5 & 4 & 3 \\
& Agent B & 3 & 4 & 5 & 6 \\
$\alpha^{(5)}$ & Agent A & 7 & 6 & 5 & 4 \\
& Agent B & 4 & 6 & 5 & 7 \\
\hline
\end{tabular}


Fig. 6 Agent A's total utility in each round with different concession adjusted coefficients

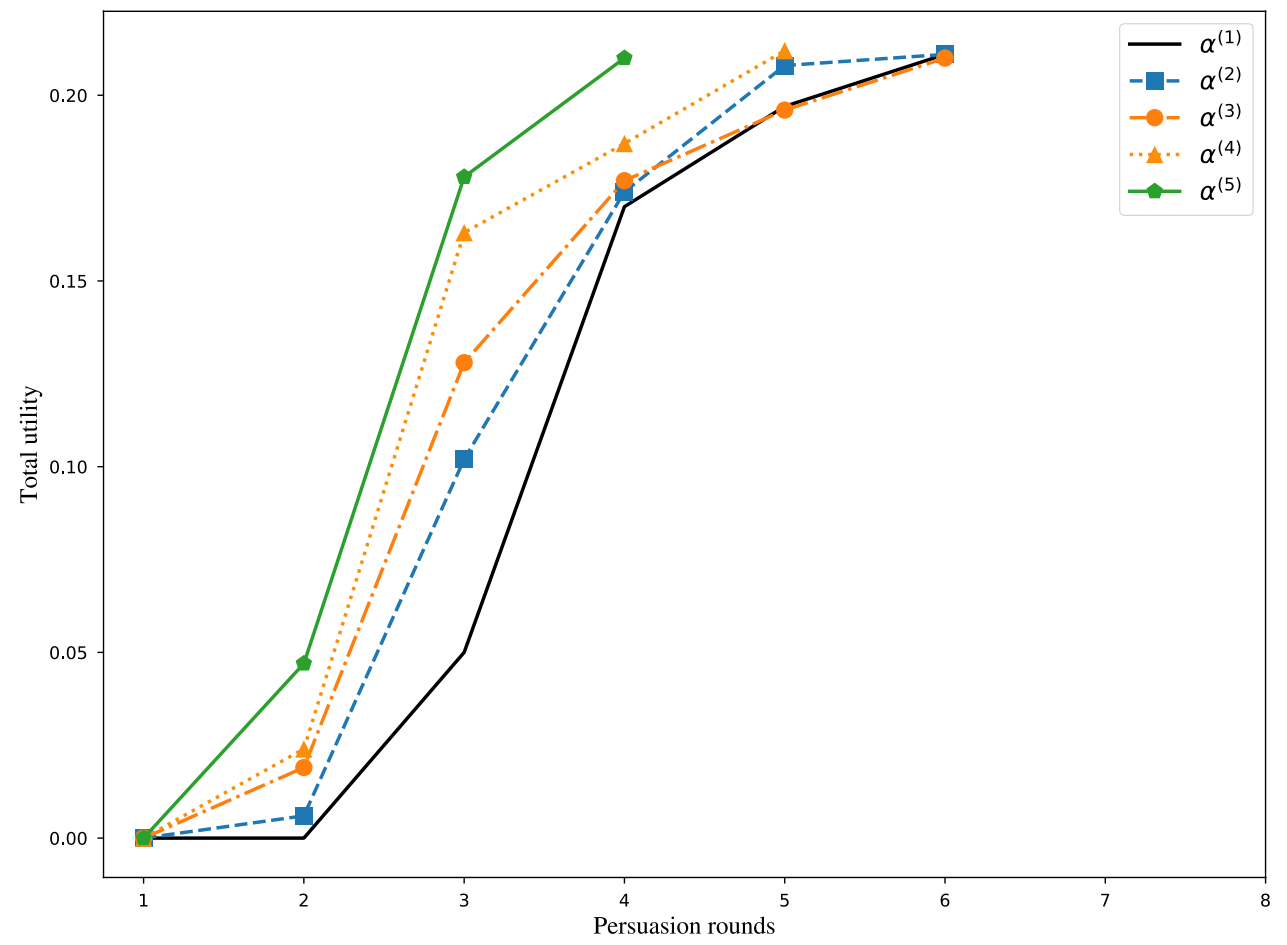

Fig. 7 Agent B's total utility in each round with different concession adjusted coefficients

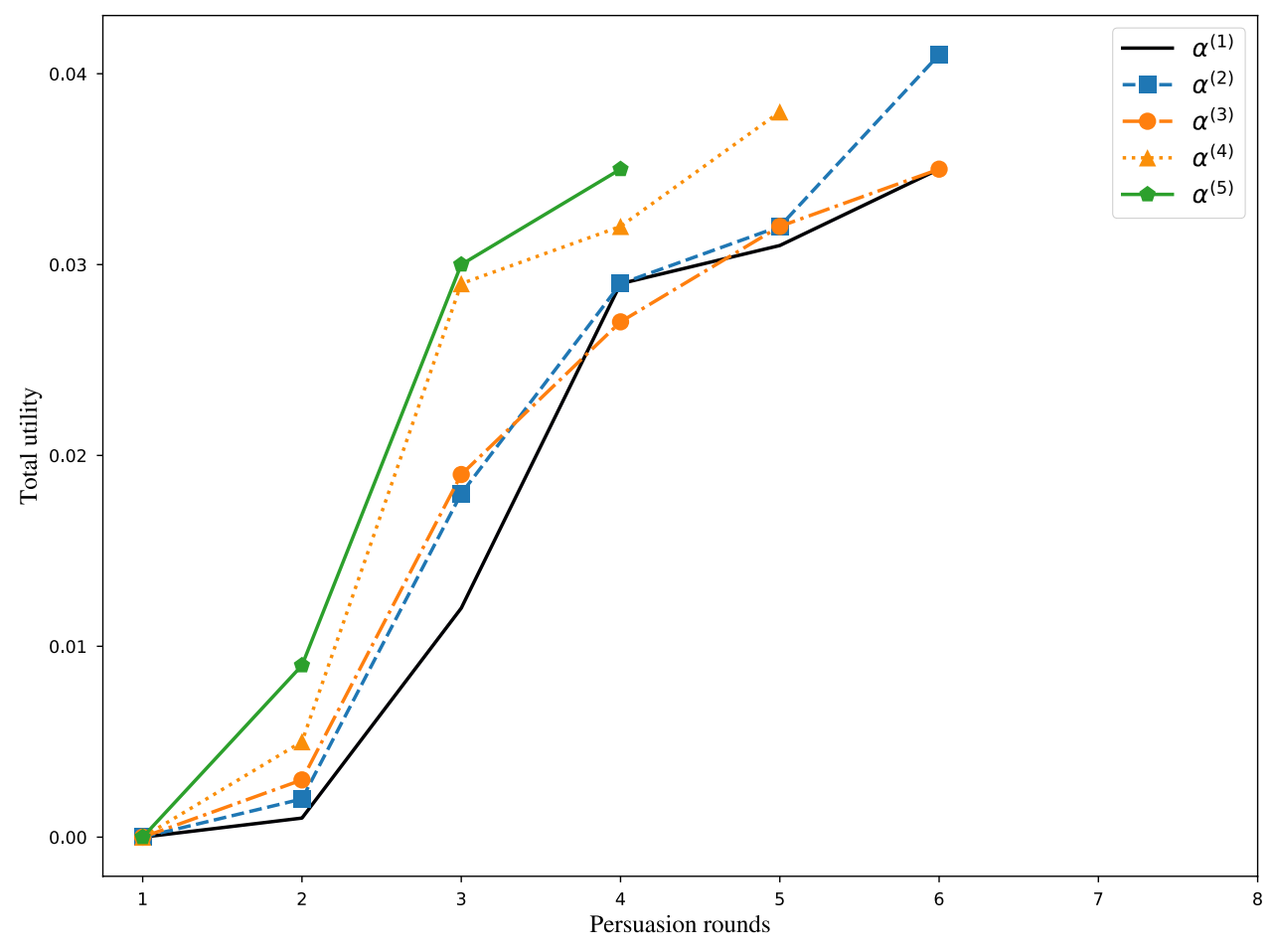

nor reputation and 9 rounds in the model considering only emotion. Second, the proposed model generally results in a better negotiation result. For instance, the agreed price is 5.8 in the proposed model, which is consistently lower than 6 in the model with neither emotion nor reputation and 6.1 in the model with only emotion. The delivery quality is 7.6 in the proposed model, which is greater than the agreed quality in the model with neither emotion nor reputation. 
Table 12 The process of Agent A's persuasion with neither emotion nor reputation

\begin{tabular}{llllll}
\hline Rounds & $\begin{array}{l}\text { Received type } \\
\text { of persuasion }\end{array}$ & \multicolumn{2}{l}{ Proposal } & Total utility \\
\cline { 3 - 5 } & & Price & Quality & Delivery time & \\
\hline 1 & & 3 & 9 & 3 & 0.000 \\
2 & Complaint & 3.8 & 8.6 & 4.2 & 0.003 \\
3 & Explanation & 4.4 & 8.3 & 5.1 & 0.071 \\
4 & Analogy & 4.8 & 8.1 & 5.7 & 0.175 \\
5 & Threat & 5.0 & 8.0 & $6^{\mathrm{a}}$ & 0.244 \\
6 & Threat & 5.2 & 7.9 & $6^{\mathrm{a}}$ & 0.254 \\
7 & Threat & 5.4 & 7.8 & $6^{\mathrm{a}}$ & 0.266 \\
8 & Threat & 5.6 & 7.7 & $6^{\mathrm{a}}$ & 0.279 \\
9 & Threat & 5.8 & 7.6 & $6^{\mathrm{a}}$ & 0.294 \\
10 & Threat & 6.0 & 7.5 & $6^{\mathrm{a}}$ & 0.312 \\
\hline
\end{tabular}

${ }^{\text {a }}$ The original value is changed to the maximum value of the negotiating issue acceptable to Agent $A$

Table 13 The process of Agent B's persuasion with neither emotion nor reputation

\begin{tabular}{llllll}
\hline Rounds & $\begin{array}{l}\text { Received type } \\
\text { of persuasion }\end{array}$ & \multicolumn{2}{l}{ Proposal } & Total utility \\
\cline { 3 - 5 } & & Price & Quality & Delivery time & \\
\hline 1 & 9 & 6 & 7 & 0.000 \\
2 & Threat & 8.4 & 6.9 & 6.2 & 0.002 \\
3 & Analogy & 7.95 & 8.1 & 5.6 & 0.018 \\
4 & Explanation & 7.65 & 8.7 & 5.2 & 0.041 \\
5 & Complaint & 7.5 & $9^{\mathrm{a}}$ & 5.0 & 0.056 \\
6 & Complaint & 7.4 & $9^{\mathrm{a}}$ & 4.8 & 0.058 \\
7 & Complaint & 7.2 & $9^{\mathrm{a}}$ & 4.6 & 0.060 \\
8 & Complaint & 7.05 & $9^{\mathrm{a}}$ & 4.4 & 0.062 \\
9 & Complaint & 6.9 & $9^{\mathrm{a}}$ & 4.2 & 0.064 \\
10 & Complaint & 6.75 & $9^{\mathrm{a}}$ & 4.0 & 0.066 \\
\hline
\end{tabular}

a The original value is changed to the maximum value of the negotiating issue acceptable to Agent $B$

Table 14 The process of Agent A's persuasion with only emotion

\begin{tabular}{llllll}
\hline Rounds & $\begin{array}{l}\text { Received type } \\
\text { of persuasion }\end{array}$ & \multicolumn{2}{l}{ Proposal } & Total utility \\
\cline { 3 - 5 } & & Price & Quality & Delivery time & \\
\hline 1 & & 9 & 3 & 0.000 \\
2 & Complaint & 3.8 & 8.6 & 4.2 & 0.003 \\
3 & Explanation & 4.4 & 8.3 & 5.1 & 0.071 \\
4 & Analogy & 4.8 & 8.1 & 5.7 & 0.175 \\
5 & Analogy & 5.2 & 7.9 & $6^{\mathrm{a}}$ & 0.254 \\
6 & Threat & 5.4 & 7.8 & $6^{\mathrm{a}}$ & 0.266 \\
7 & Threat & 5.6 & 7.7 & $6^{\mathrm{a}}$ & 0.279 \\
8 & Threat & 5.8 & 7.6 & $6^{\mathrm{a}}$ & 0.294 \\
9 & Threat & 6.0 & 7.5 & $6^{\mathrm{a}}$ & 0.312 \\
\hline
\end{tabular}

a The original value is changed to the maximum value of the negotiating issue acceptable to Agent A
Table 15 The process of Agent B's persuasion with only emotion

\begin{tabular}{llllll}
\hline Rounds & $\begin{array}{l}\text { Received type } \\
\text { of persuasion }\end{array}$ & \multicolumn{2}{l}{ Proposal } & Total utility \\
\cline { 3 - 5 } & & Price & Quality & Delivery time \\
\hline 1 & 9 & 6 & 7 & 0.000 \\
2 & Threat & 8.4 & 7.2 & 6.2 & 0.002 \\
3 & Analogy & 7.95 & 8.1 & 5.6 & 0.018 \\
4 & Explanation & 7.65 & 8.7 & 5.2 & 0.041 \\
5 & Explanation & 7.35 & $9^{\mathrm{a}}$ & 4.8 & 0.058 \\
6 & Complaint & 7.2 & $9^{\mathrm{a}}$ & 4.6 & 0.060 \\
7 & Complaint & 7.05 & $9^{\mathrm{a}}$ & 4.4 & 0.062 \\
8 & Complaint & 6.9 & $9^{\mathrm{a}}$ & 4.2 & 0.064 \\
9 & Complaint & 6.75 & $9^{\mathrm{a}}$ & 4.0 & 0.066 \\
\hline
\end{tabular}

${ }^{\text {a }}$ The original value is changed to the maximum value of the negotiating issue acceptable to Agent $B$

\section{Conclusions}

\subsection{Discussion}

This paper proposes an emotion-adjusted concession model for agent-based negotiation. This model incorporates an agent's emotion which reflects the agent's response to a rival's proposal into the selection of a persuasion type and the calculation of concessions. As such, an agent can better simulate decision-making behavior of human beings in negotiation; accordingly, the automated negotiation systems with such emotional agents have great potentials in human-to-agent automated negotiation. In sum, this paper makes efforts in the following aspects.

This paper proposes a method to quantify emotions in the setting of agent-based negotiation. Emotions are modeled as an agent's response to the difference between its expected issue value and the value from the rival's proposal. The greater the absolute value of the difference, the stronger an agent's emotions. The emotion is a mental state of a human being. Via the proposed quantification method of emotions, not only an agent's response to external stimuli can be internalized to the agent's mental state (i.e., emotions), but also a wide range of emotions can be captured and measured. Accordingly, an agent can be made more capable of behaving like a real human in human-toagent negotiation which requires the software agent to be as equivalent as possible to its human counterpart.

This paper establishes a connection between emotions and persuasion types. We identify four types of persuasion that an agent can use to guide its calculation of concessions and relate each type of persuasion to emotions. Emotions play a significant role in affecting persuasion behavior in human-conducted negotiation. The established connection proposes a way to characterize how emotions can affect an agent's persuasion type and accordingly an 
agent's selection of a persuasion type can be driven by the agent's internalized state rather than merely external information. Thereby, an agent is enabled to make concessions more adaptive to the negotiation progress, enhancing the quality of an agent's interactions with human counterparts.

This paper investigates the effects of reputation on an agent's evaluation of a proposal. Reputation has been recognized as an important factor to select a trustworthy negotiating partner. This paper goes a further step by considering the effects of reputation in the process of an agent's negotiation. An agent's evaluation of a proposal is not only affected by the issues contained in the proposal but also influenced by the rival agent's reputation. As such, an agent is able to select distinct persuasion behavior in terms of a rival agent's reputation.

\subsection{Limitations and future research}

Notwithstanding those achievements, this research has some limitations. First, this research attempts to make initial efforts towards modeling the effects of emotions into agent-based negotiation and thus uses a relatively simplified criterion to map emotions to the types of persuasion. This connection is critical to increase the adaptive level of concessions made by an agent and therefore future research can explore more objective criteria to establish this connection.

Second, we consider the effects of reputation at the beginning of negotiation. However, an agent's reputation will change during negotiation and will inflict additional effects on an agent's concession-making in addition to the initial belief in the rival agent. For example, an agent's changed reputation level may alter the credibility of the agent's proposal. Future research can further considers these dynamic effects of reputation.

Last, this research aims to construct an agent-based concession model that considers an agent's emotion. We provide a numerical example to illustrate the procedures and the advantages of the proposed model. Its main purpose is to test the feasibility of the model, but we have not examined the model in a real case. In spite of this limitation, our proposed method to quantify emotion and to relate emotion to the persuasion type is relatively general. Once a sufficient historical data of negotiations in an industry can be collected, the cut-off points of emotion and the base concessions as well as adjusted coefficients can be more reliably estimated. Thus, future research can further verify this model and explore its characteristics in more practical scenarios.

Funding This research is partially supported by the National Natural Science Foundation of China $(71972177,71502170)$ and the Fundamental Research Funds for the Central Universities of China (2020YJSGL04)

\section{References}

Akhgar B, Salahi Parvin E, Sherkat MH (2014) Axiomatic agent based architecture for agile decision making in strategic information systems. J Ambient Intell Hum Comput 5(1):93-104. https://doi. org/10.1007/s12652-012-0136-9

Amgoud L, Prade H (2004) Generation and evaluation of different types of arguments in negotiation. In: Proc. of the international workshop on non-monotonic reasonin, Whistler BC, Canada, pp $10-15$

Basheer GS, Ahmad MS, Tang AY, Graf S (2015) Certainty, trust and evidence: towards an integrative model of confidence in multiagent systems. Comput Hum Behavior 45:307-315. https://doi. org/10.1016/j.chb.2014.12.030, http://www.sciencedirect.com/ science/article/pii/S074756321400747X

Bradley MM, Lang PJ (1994) Measuring emotion: The self-assessment manikin and the semantic differential. J Behavior Therapy Exper Psychiatry 25(1):49-59. https://doi.org/10.1016/0005-7916(94) 90063-9, http://www.sciencedirect.com/science/article/pii/00057 91694900639

Cao M, Luo X, Luo XR, Dai X (2015) Automated negotiation for e-commerce decision making: a goal deliberated agent architecture for multi-strategy selection. Decision Support Syst 73:1-14. https://doi.org/10.1016/j.dss.2015.02.012, http://www.sciencedir ect.com/science/article/pii/S0167923615000329

Castellanos S, Rodrguez LF, Castro LA, Gutierrez-Garcia JO (2018) A computational model of emotion assessment influenced by cognition in autonomous agents. Biol Inspired Cogn Architect 25:26-36. https://doi.org/10.1016/j.bica.2018.07.007, http://www. sciencedirect.com/science/article/pii/S2212683X18300744

Chen Py, Li Y1 (2010) Multi-attribute automatic negotiation model based on niche genetic algorithm. In: 2010 Chinese Control and Decision Conference, pp 3380-3384. https://doi.org/10.1109/ CCDC.2010.5498579

Creed C, Beale R, Cowan B (2014) The impact of an embodied agent's emotional expressions over multiple interactions. Interact Comput 27(2):172-188. https://doi.org/10.1093/iwc/iwt064, https://acade mic.oup.com/iwc/article-pdf/27/2/172/1890261/iwt064.pdf

Fershtman C, Judd KL (1987) Equilibrium incentives in oligopoly. Am Econ Rev 77(5):927-940. http://www.jstor.org/stable/1810218

Frijda NH (1988) The laws of emotion. Am Psychol 43:349-58

Hajimiri MH, Nili Ahmadabadi M, Rahimi-Kian A (2014) An intelligent negotiator agent design for bilateral contracts of electrical energy. Expert Syst Appl 41(9):4073- 4082. https://doi.org/10. 1016/j.eswa.2013.12.034, http://www.sciencedirect.com/science/ article/pii/S0957417413010099

Jain P, Dahiya D (2012) An Intelligent Multi Agent Framework for E-commerce Using Case Based Reasoning and Argumentation for Negotiation. In: Dua S, Gangopadhyay A, Thulasiraman P, Straccia U, Shepherd M, Stein B (eds) Information systems, technology and management, Springer, Berlin, Heidelberg, Communications in Computer and Information Science, pp 164-175. Doi: https:// doi.org/10.1007/978-3-642-29166-1_15

Kolic B, Dyer J (2020) Data-driven modeling of public risk perception and emotion on twitter during the covid-19 pandemic. 2008.00854

Kowalczyk R, Phiong V, Dunstall S, Owens B (2004) Towards supporting collaborative scheduling in adaptive supply networks with negotiation agents. J Decis Syst 13(4):441-460. https://doi.org/10. 3166/jds.13.441-460

Kraus S, Sycara K, Evenchik A (1998) Reaching agreements through argumentation: a logical model and implementation. Artif Intell 104(1):1-69. https://doi.org/10.1016/S0004-3702(98)00078-2, http://www.sciencedirect.com/science/article/pii/S000437029 8000782 
Leary K, Julianna P, Wheeler M (2013) Negotiating with emotion. Harvard Business Rev 91(1/2):96-103

Louta M, Roussaki I, Pechlivanos L (2008) An intelligent agent negotiation strategy in the electronic marketplace environment. Eur J Operat Res 187(3):1327-1345. https://doi.org/10.1016/j.ejor. 2006.09.016

Maria KA, Zitar RA (2007) Emotional agents: a modeling and an application. Inf Softw Technol 49(7):695-716. https://doi.org/10. 1016/j.infsof.2006.08.002, http://www.sciencedirect.com/science/ article/pii/S0950584906001030

Martinovski B (2010) Emotion in Negotiation, Springer Netherlands, Dordrecht, pp 65-86. https://doi.org/10.1007/ 978-90-481-9097-3_5

Mehrabian A (1996) Analysis of the big-five personality factors in terms of the PAD temperament model. Austr J Psychol 48(2):86. https://doi.org/10.1080/00049539608259510

Meyer JC (2006) Reasoning about emotional agents. Int J Intell Syst 21(6):601-619

Mian SQ, Oinas-Kukkonen H (2016) An analysis of previous information systems research on emotion. In: Proceedings of the fourth international workshop on behavior change support systems colocated with the 11th International Conference on Persuasive Technology, Salzburg, Austria, vol 1573, pp 36-55

Moerland TM, Broekens J, Jonker CM (2018) Emotion in reinforcement learning agents and robots: a survey. Machine Learn 107(2):443-480. https://doi.org/10.1007/s10994-017-5666-0

Monteserin A, Amandi A (2013) A reinforcement learning approach to improve the argument selection effectiveness in argumentationbased negotiation. Expert Syst Appl 40(6):2182-2188. https://doi. org/10.1016/j.eswa.2012.10.045, http://www.sciencedirect.com/ science/article/pii/S0957417412011694

Pan L, Luo X, Meng X, Miao C, He M, Guo X (2013) A two-stage winwin multiattribute negotiation model: optimization and then concession. Comput Intell 29(4):577-626. https://doi.org/10.1111/j. 1467-8640.2012.00434.x, https://onlinelibrary.wiley.com/doi/abs/ 10.1111/j.1467-8640.2012.00434.x

Paradeda RB, Ferreira MJ, Dias J, Paiva A (2017) How robots persuasion based on personality traits may affect human decisions. In: Proceedings of the companion of the 2017 ACM/IEEE international conference on human-robot interaction, Association for Computing Machinery, New York, NY, USA, HRI 17, pp 251252. Doi: https://doi.org/10.1145/3029798.3038348 (event-place: Vienna, Austria tex.numpages: 2)

Ramezani F, Ghasem-Aghaee N, Kazemifard M (2011) Modeling of emotional-social negotiator agents. In: 2011 11th international conference on intelligent systems design and applications, pp 42-46. Doi: https://doi.org/10.1109/ISDA.2011.6121628

Ruijten PAM, Midden CJH, Ham J (2016) Ambiguous agents: the influence of consistency of an artificial agents social cues on emotion recognition, recall, and persuasiveness. Int J Human Comput Interaction 32(9):734-744. https://doi.org/10.1080/10447318. 2016.1193350

Salgado M, Clempner JB (2018) Measuring the emotional state among interacting agents: a game theory approach using reinforcement learning. Expert Syst Appl 97:266-275. https://doi.org/10.1016/j. eswa.2017.12.036, http://www.sciencedirect.com/science/article/ pii/S095741741730859X
Santos R, Marreiros G, Ramos C, Neves J, Bulas-Cruz J (2011) Personality, emotion, and mood in agent-based group decision making. IEEE Intell Syst 26(6):58-66. https://doi.org/10.1109/MIS.2011. 92 (conference Name: IEEE Intelligent Systems)

Sengul M, Gimeno J, Dial J (2012) Strategic delegation: a review, theoretical integration, and research agenda. J Manag 38(1):375-414. https://doi.org/10.1177/0149206311424317

Subagdja B, Tan AH, Kang Y (2019) A coordination framework for multi-agent persuasion and adviser systems. Expert Syst Appl 116:31-51. https://doi.org/10.1016/j.eswa.2018.08.030

Sun H, Wu J, Huang T, He G (2014) A concession model based on agent persuasion classification and its implementation. Oper Res Manag Sci 6:252-256

Sycara KP (1990) Persuasive argumentation in negotiation. Theory Decis 28(3):203-242. https://doi.org/10.1007/BF00162699

Van Kleef GA, De Dreu CK, Pietroni D, Manstead ASR (2006) Power and emotion in negotiation: power moderates the interpersonal effects of anger and happiness on concession making. Eur J Soc Psychol 36(4):557-581. https://doi.org/10.1002/ejsp.320, https:// onlinelibrary.wiley.com/doi/abs/10.1002/ejsp.320

Vickers J (1985) Delegation and the theory of the firm. Econ J 95:138147. http://www.jstor.org/stable/2232877

Wang F, Zhuo X, Niu B, He J (2017) Who canvasses for cargos? incentive analysis and channel structure in a shipping supply chain. Transport Res Part B Methodol 97:78-101. https://doi.org/10. 1016/j.trb.2016.11.007, http://www.sciencedirect.com/science/ article/pii/S0191261516302934

Wang Y, Lucas G, Khooshabeh P, de Melo C, Gratch J (2015) Effects of emotional expressions on persuasion. Soc Influence 10(4):236 249. https://doi.org/10.1080/15534510.2015.1081856

Whitford AB, Bottom WP, Miller GJ (2013) The (negligible) benefit of moving first: efficiency and equity in principal-agent negotiations. Group Decision Negot 22(3):499-518. https://doi.org/10. 1007/s10726-011-9280-4

Wong T, Fang F (2010) A multi-agent protocol for multilateral negotiations in supply chain management. Int J Prod Res 48(1):271-299. https://doi.org/10.1080/00207540802425393

Xue X, Shen Q, Li H, O'Brien WJ, Ren Z (2009) Improving agentbased negotiation efficiency in construction supply chains: a relative entropy method. Autom Constr 18(7):975-982. https://doi. org/10.1016/j.autcon.2009.05.002, http://www.sciencedirect.com/ science/article/pii/S092658050900065X

Yu C, Wong TN (2015) An agent-based negotiation model for supplier selection of multiple products with synergy effect. Expert Syst Appl 42(1):223-237. https://doi.org/10.1016/j.eswa.2014.07.057, http:// www.sciencedirect.com/science/article/pii/S0957417414004734

Zhang L, Song H, Chen X, Hong L (2011) A simultaneous multiissue negotiation through autonomous agents. Eur J Oper Res 210(1):95-105. https://doi.org/10.1016/j.ejor.2010.10.011, http:// www.sciencedirect.com/science/article/pii/S0377221710006521

Publisher's Note Springer Nature remains neutral with regard to jurisdictional claims in published maps and institutional affiliations. 